\title{
Species-specific inhibition of fungal protein synthesis by sordarin: identification of a sordarin-specificity region in eukaryotic elongation factor 2
}

Department of Animal Health, Merck Research Laboratories, PO Box 2000, Rahway, NJ 07065, USA

\author{
Mythili Shastry, Jennifer Nielsen, Theresa Ku, Ming-Jo Hsu, \\ Paul Liberator, Jennifer Anderson, Dennis Schmatz \\ and Michael C. Justice
}

Author for correspondence: Michael C. Justice. Tel: +1 732594 3941. Fax: +1 7325946708. e-mail:michael_justice@merck.com

The sordarin class of natural products selectively inhibits fungal protein synthesis by impairing the function of eukaryotic elongation factor 2 (eEF2). Mutations in Saccharomyces cerevisiae eEF2 or the ribosomal stalk protein rpPO can confer resistance to sordarin, although eEF2 is the major determinant of sordarin specificity. It has been shown previously that sordarin specifically binds $S$. cerevisiae eEF2 while there is no detectable binding to eEF2 from plants or mammals, despite the high level of amino acid sequence conservation among these proteins. In both whole-cell assays and in vitro translation assays, the efficacy of sordarin varies among different species of pathogenic fungi. To investigate the basis of sordarin's fungal selectivity, eEF2 has been cloned and characterized from several sordarin-sensitive and -insensitive fungal species. Results from in vivo expression of Candida species eEF2s in S. cerevisiae and in vitro translation and growth inhibition assays using hybrid $S$. cerevisiae eEF2 proteins demonstrate that three amino acid residues within eEF2 account for the selectivity of this class of compounds. It is also shown that the corresponding residues at these positions in human eEF2 are sufficient to confer sordarin insensitivity to $S$. cerevisiae identical to that observed with mammalian eEF2.

Keywords: translation, elongation, sordarin specificity, protein synthesis

\section{INTRODUCTION}

Eukaryotic elongation factor 2 (eEF2) catalyses the movement of the ribosome along the mRNA and is essential for translocation of peptidyl tRNA from the A to $\mathrm{P}$ site of the ribosome. eEF2 is a member of the GTPase superfamily of proteins and it is presumed that translocation is driven by a conformational change in eEF2 accompanied by a rapid hydrolysis of GTP. Our previous work has shown that the natural product sordarin and its derivatives specifically inhibit fungal translation by binding to and stabilizing the eEF2GDP-ribosome complex in Saccharomyces cerevisiae

Abbreviations: eEF2, eukaryotic elongation factor 2; EF-G, prokaryotic elongation factor $\mathrm{G}$.

The GenBank accession numbers for the sequences reported in this manuscript are AF107286-AF107291, AF292693 and AF248644.
(Justice et al., 1998). Recent studies by other workers suggest that sordarin prevents the transition of the ribosome from the PRE-eEF2-GTP to POST-eEF2GTP state in Candida albicans (Dominguez et al., 1999). Despite the extensive homology between the eEF2s, there is no significant binding of sordarin to either plant or mammalian eEF2 (Dominguez et al., 1998; Justice et al., 1998), thus making sordarin a fungal-specific inhibitor of translation. Although eEF2 by itself can bind sordarin weakly, the ribosome is essential for highaffinity binding (Dominguez \& Martin, 1998; Justice et al., 1998, 1999). Moreover, genetic studies have revealed that mutations in S. cerevisiae eEF2 or the ribosomal stalk protein $\mathrm{rpP} 0$ can confer resistance to sordarin although eEF2 is the principal determinant of sordarin specificity (Capa et al., 1998; Gomez-Lorenzo \& GarciaBustos, 1998; Justice et al., 1998, 1999).

The sordarins display varying levels of antifungal 
activity when tested against different species of pathogenic fungi (Dominguez et al., 1998; Herreros et al., 1998). For example, sordarin and its glycone-substituted derivatives inhibit growth of species such as $\mathrm{Ca}$. albicans, Candida glabrata, Candida tropicalis and Cryptococcus neoformans, while other species including Candida parapsilosis and Candida krusei are insensitive to high levels of these compounds. The activity of the sordarins has been demonstrated in whole-cell assays and in cell-free translation systems, consistent with the role of these compounds as protein synthesis inhibitors (Dominguez et al., 1998).

In an effort to understand the molecular basis for the fungal specificity of sordarin and its selectivity amongst different species of fungi, we have cloned and characterized the eEF2s from various sordarin-sensitive and -insensitive species. Our results show that amino acid residues at three specific positions in fungal eEF2 are primarily responsible for the observed differences in sordarin's spectrum. Moreover, we demonstrate that the corresponding residues in mammalian eEF2 are sufficient to confer a sordarin-insensitive phenotype to $S$. cerevisiae eEF2 and therefore account for sordarin's fungal specificity.

\section{METHODS}

Yeast strains, plasmids and compounds. Sordarin was isolated essentially as described from Sordaria arenosa (Hauser \& Sigg, 1971) and preparation of the sordarin analogue L793,422 has been previously described (Justice et al., 1998). The sordarin-sensitive strain YEFD12h, harbouring the plasmids $\mathrm{pURA3-EFT1} \mathrm{or} \mathrm{YCpEFT2} \mathrm{for} \mathrm{viability} \mathrm{and} \mathrm{heterologous}$ expression assays are described elsewhere (Justice et al., 1998; Phan et al., 1993). The genes encoding the heterologous eEF2 were cloned into YCplac111 and transformed into strain

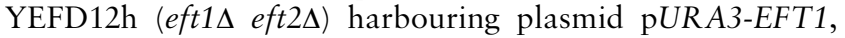
and leucine prototrophs were selected. These transformants were subsequently plated on medium containing 5-fluoroorotic acid, where growth indicates loss of the pURA3-EFT1 plasmid and thus demonstrates functional complementation. Standard yeast genetic methods and media including synthetic complete (SC) and synthetic media without leucine (SC-Leu) and YPAD medium were employed. Growth inhibition assays were performed as described previously (Justice et al., 1998).

Cloning and expression of fungal EFT2 genes. The EFT2 genes encoding eEF2 from Candida species were cloned by PCR using synthetic oligonucleotides that were designed based on the published ORF sequence of $\mathrm{Ca}$. albicans EFT2 (GenBank entry Y09664). The 5' primer contains a DraI restriction enzyme recognition sequence and the $3^{\prime}$ primer has $X h o I$ and $M l u I$ sites for subcloning purposes (oligonucleotide sequences for cloning and modification of the genes encoding the eEF2 proteins described in this manuscript are available on request). The $\mathrm{Ca}$. glabrata EFT2 gene was cloned using primers based on the ORF of the $S$. cerevisiae EFT2 gene.

Genomic DNA from Ca. glabrata, Candida guilliermondii, Ca. krusei, Candida lusitaniae, Ca. parapsilosis and Ca. tropicalis species was prepared by standard procedures and purified with a QIAamp Tissue Kit (Qiagen) according to the manufacturer's specifications. PCR products were amplified from genomic DNA with the synthetic oligonucleotides described above using Klentaq polymerase (Clontech) according to the manufacturer's recommendations.

At least three independent PCR reaction products were obtained from genomic DNA from each of the Candida species. Following initial sequence identification of three identical EFT2 PCR products from each fungal species, the products were cloned into the vector pGEM-T Easy (Promega), followed by transformation into the Escherichia coli strain DH5 $\alpha$ (Life Technologies). Plasmid DNA was prepared with Qiaspin columns (Qiagen) according to the manufacturer's instructions and digested with appropriate restriction enzymes to verify the identity of the constructs. At least four clones of each of the Candida eEF2 genes in pGEMT Easy were sequenced with an ABI Prism 377 DNA Sequencer according to the manufacturer's recommendations (PE-Applied Biosystems). Sequences were analysed using Sequencher DNA analysis software (Gene Codes). These sequence data were compared with the original sequence obtained from the PCR products to verify the identity of each Candida species EFT2 obtained. The Candida EFT2 genes were excised as 5'DraI to 3'-XhoI fragments from the pGEM-T Easy plasmids, gel-purified using QIAEX (Qiagen) and ligated into the expression vector YCpEFT2 (Justice et al., 1998) digested with HpaI and XboI. The final constructs contained the Candida species EFT2 genes expressed from the $S$. cerevisiae EFT2 promoter. All of the EFT2 clones described above contain about 25 nucleotides of PCR primer sequence at the $5^{\prime}$ and $3^{\prime}$ ends of the Candida species eEF2s and include the start and stop codons that were derived from either $\mathrm{Ca}$. albicans EFT2 (Ca. Insitaniae, Ca. parapsilosis and Ca. tropicalis) or S. cerevisiae EFT2 (Ca. glabrata). The fungal EFT2 constructs were transformed into the $S$. cerevisiae $\Delta$ eft $1 \Delta e f t 2$ deletion strain YEFD12h. Transformants were selected for leucine prototrophy and subsequently plated on 5-fluoroorotic acid agar medium to select strains that have lost pURA3-EFT1 that encodes $S$. cerevisiae eEF2 while retaining the plasmid encoding the heterologous eEF2. For Ca. guilliermondii and Ca. krusei, primers were designed based on the consensus sequence of a Candida species EFT2 nucleotide alignment. These were used to amplify a region corresponding to nucleotides 1400-1800 in the gene encoding eEF2 with the corresponding genomic DNA as a template. These PCR products were sequenced, cloned and verified as described above. The Candida EFT2 sequences described in this paper were submitted to GenBank (AF107286-AF107291 and AF292693). The deduced full-length amino acid sequences of the eEF2s were aligned using the CLUSTAL w program (Thompson et al., 1994).

Full-length cDNA clones encoding the Cr. neoformans EFT2 homologue were isolated from a lambda ZAP II cDNA library (NIH AIDS Research and Reference Reagent Program catalogue number 2077, contributed by Dr Jeffrey Edman). Approximately $10^{6}$ p.f.u. from the amplified library were screened at reduced stringency $(40 \%$ formamide $/ 5 \times \mathrm{SSPE} /$ $35^{\circ} \mathrm{C}$ ) using a gel-purified $1.9 \mathrm{~kb}$ HindIII fragment from the protein coding region of $S$. cerevisiae EFT2 as hybridization probe. Twenty hybridization-positive clones were plaquepurified, subcloned by in vivo excision to pBluescript SK (Stratagene) and characterized. Seventeen clones had inserts approximately $2.7 \mathrm{~kb}$ in length; several of these were sequenced and the sequence submitted to GenBank (AF248644). The ORF of the Cr. neoformans EFT2 gene was modified for expression in $S$. cerevisiae by PCR. The $5^{\prime}$ primer introduced a HpaI site for cloning and appended a portion of the $S$. cerevisiae EFT2 5'-untranslated region to the $\mathrm{Cr}$. neoformans protein coding region and destroyed a $\mathrm{HpaI}$ site at the $5^{\prime}$ end 
of the Cr. neoformans gene. The 3' primer introduced an MluI site for subcloning. The product of the PCR reaction was cloned, sequenced to confirm PCR accuracy and ligated to YCpEFT2 digested with HpaI and MluI. As described above, this construct was then transformed and assayed for function in the S. cerevisiae strain YEFD12h.

Construction of substituted S. cerevisiae eEF2s. Site-directed mutagenesis of the $\mathrm{Ca}$. parapsilosis gene encoding eEF2 to create S521Y and N523S single substitution mutants and the S521Y N523S doubly substituted mutant was accomplished by the PCR overlap extension technique (Ho et al., 1989). Modified S. cerevisiae eEF2 genes encoding amino acid residues from Ca. glabrata, Ca. guilliermondii, Ca. krusei, Ca. lusitaniae, Ca. parapsilosis, Cr. neoformans or human eEF2 were constructed by cassette mutagenesis as described by Prodromou \& Pearl (1992).

Plasmid DNA from each fungal EFT2 construct was used to transform the $S$. cerevisiae $\Delta$ eft 1 eft 2 deletion strain YEFD12h to leucine prototrophy and strains harbouring only the fungal EFT2 constructs were selected as described above. $S$. cerevisiae strains containing the fungal EFT2 constructs were also assayed for the predicted sordarin-resistance phenotype by plating cells on medium containing sordarin at 5 or $10 \mu \mathrm{g} \mathrm{ml}^{-1}$. Plates were incubated at $29^{\circ} \mathrm{C}$ for approximately $3 \mathrm{~d}$ until colonies appeared. Growth inhibition assays to generate dose response curves were performed as previously described with individual assays performed at least three times (Justice et al., 1998).

Biochemical assays. S. cerevisiae post-ribosomal extracts were prepared from cultures grown to mid-exponential phase in YPAD medium as described previously (Justice et al., 1999). The potency of sordarin was assessed in in vitro translation systems as described previously (Justice et al., 1998, 1999), using $0.05 A_{260}$ of wild-type ribosomes and $3 \mu \mathrm{g} \mathrm{S}$. cerevisiae post-ribosomal extract for each $12.5 \mu \mathrm{l}$ assay. Binding studies with a labelled sordarin derivative and unlabelled sordarin (Justice et al., 1998) were employed to establish the lower limit of sordarin sensitivity using approximately $0 \cdot 25$ pmol eEF2 per assay.

\section{RESULTS}

\section{Cloning and heterologous expression of fungal EFT2 genes}

Previous studies have demonstrated that eEF2, the product of the $S$. cerevisiae EFT1 and EFT2 genes, is the primary molecular target of sordarin and its analogues (Capa et al., 1998; Justice et al., 1998). Other workers have shown that sordarin and its derivatives inhibit translation elongation in sensitive fungi such as $\mathrm{Ca}$. albicans and $\mathrm{Ca}$. glabrata, while having no effect on naturally insensitive species like Ca. parapsilosis and Ca. krusei (Dominguez et al., 1998; Herreros et al., 1998). These findings are consistent with our results from in vitro translation assays reconstituted with eEF2 from other fungal species. For example, sordarin inhibits elongation when the assay is reconstituted with $\mathrm{Ca}$. albicans, Ca. glabrata and Cr.neoformans eEF2, but has no effect in elongation assays supported by $\mathrm{Ca}$. parapsilosis, Ca. lusitaniae or Ca. krusei eEF2 (Fig. 1). Taken together, these results suggest that the differential sensitivity to sordarin among fungal species can be attributed specifically to eEF2.

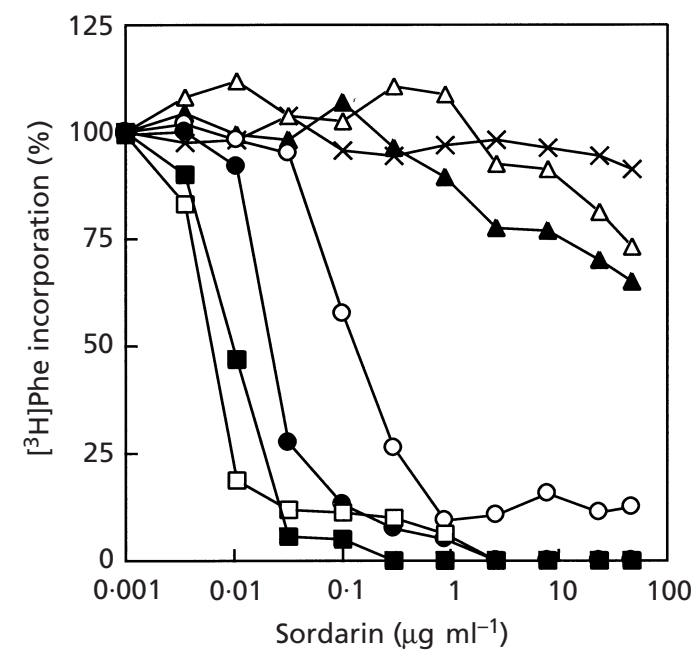

Fig. 1. Soluble translation factors derived from pathogenic fungi and ribosomes from $S$. cerevisiae confer a range of sordarin sensitivity. Translation assays were performed as described in Methods using ribosomes from S. cerevisiae and post-ribosomal extracts as follows: $\square, S$. cerevisiae; $\square, \mathrm{Ca}$. albicans; O, Ca. glabrata; O, Cr. neoformans; $\mathbf{\Delta}, \mathrm{Ca}$. parapsilosis; $\triangle$, Ca. lusitaniae; $\times$, Ca. krusei.

Table 1. Heterologous expression of Candida species eEF2s in S. cerevisiae

S. cerevisiae strains expressing full-length heterologous eEF2s under the $S$. cerevisiae EFT2 promoter were generated as described in Methods. $\mathrm{IC}_{50}$ values were determined from growth inhibition assays in which cells were inoculated in SC medium containing sordarin serially diluted twofold from 100 to $0 \cdot 2 \mu \mathrm{g} \mathrm{ml}^{-1}$, followed by incubation at $29^{\circ} \mathrm{C}$ for approximately $16 \mathrm{~h}$. In vitro translation assays were performed as described in Methods. The $\mathrm{IC}_{50}$ values are the mean of three independent experiments. The lower limit of sensitivity is about $0.005 \mu \mathrm{g} \mathrm{ml} l^{-1}$, as noted in Methods.

\begin{tabular}{|lcc|}
\hline Source of eEF2 & \multicolumn{2}{c|}{ Sordarin $\mathrm{IC}_{\mathbf{5 0}}\left(\boldsymbol{\mu \mathrm { g } \mathrm { ml } ^ { - 1 } )}\right.$} \\
\cline { 2 - 3 } & Growth inhibition & In vitro translation \\
\hline S. cerevisiae & $0 \cdot 50$ & $0 \cdot 006$ \\
Ca. albicans & $0 \cdot 12$ & $0 \cdot 007$ \\
Ca. glabrata & $4 \cdot 00$ & $0 \cdot 025$ \\
Ca. lusitaniae & $>100$ & $>10$ \\
Ca. parapsilosis & $>100$ & $>10$ \\
\hline
\end{tabular}

To investigate the molecular basis of sordarin's specificity for fungal eEF2, we have cloned and obtained sequence data for genes encoding eEF2 from $C a$. albicans, Ca. glabrata, Ca. lusitaniae, Ca. parapsilosis, $\mathrm{Ca}$. tropicalis and $\mathrm{Cr}$. neoformans as described in Methods. A heterologous expression system was established in $S$. cerevisiae to examine the effects of sordarin on $S$. cerevisiae harbouring a foreign eEF2. With the exception of Ca.tropicalis and Cr.neoformans, each of the cloned eEF2s was able to functionally 


S.cerevisiae
C.albicans
C.tropicalis
C.glabrata
C.parapsilosis
C.lusitaniae
C.guilliermondi
C. krusei
C.neoformans
human

VEGLKRLSKSDP

VEGLKRLSKSDP

VEGLKRLSKSDP

VEGLKRLSKSDP

VEGLKRLSKSDP

VEGLKRLSKSDP

VEGLKRLSKSDP

VEGLKRLSKSDP

VEGLKRLSKSDP

VEGLKRLAKSDP
Fig. 2. Partial alignment of the deduced amino acid sequence of eEF2 from $S$. cerevisiae with fungal and human eEF2s. Fungal genes encoding eEF2s were cloned as described in Methods. The deduced amino sequences of human and fungal eEF2s were aligned using the CLUSTAL W program (Thompson et al., 1994). The 'sordarinspecificity region' corresponding to residues 517-524 of S. cerevisiae eEF2 is enclosed in the box. complement $S$. cerevisiae eEF2, although all the strains expressing Candida species EF2 had a slightly reduced growth rate with less than a twofold increase in doubling time. The ability of various Candida eEF2s to confer sordarin resistance on the host $S$. cerevisiae strain was tested as described by Justice et al. (1998). S. cerevisiae strains expressing eEF2 from Ca. glabrata, Ca. lusitaniae and $\mathrm{Ca}$. parapsilosis exhibited sordarin-resistance phenotypes paralleling those of the native organisms (Table 1). As expected, a strain of $S$. cerevisiae dependent upon $\mathrm{Ca}$. albicans eEF2 was sensitive to sordarin at concentrations similar to wild-type $S$. cerevisiae.

\section{Identification of a sordarin-specificity region within eEF2}

To determine the amino acid residues in eEF2 responsible for sordarin resistance in the Candida species, we compiled and aligned the deduced amino acid sequences of the eEF2 proteins. This alignment reveals greater than $90 \%$ identity between eEF2s from the various Candida species and approximately $85 \%$ identity with $S$. cerevisiae eEF2. A partial alignment is shown in Fig. 2. In our previous studies with sordarininsensitive $S$. cerevisiae eEF2 mutants, one-third $(5 / 15)$ of the mutants had substitutions at amino acid residues Y521 and S523 (Justice et al., 1998). The alignments in Fig. 2 showed that eEF2s from the fully sordarininsensitive $\mathrm{Ca}$. parapsilosis and $\mathrm{Ca}$. Lusitaniae have Ser and Asn at positions 521 and 523, respectively, while the eEF2 from sordarin-sensitive $\mathrm{Ca}$. albicans has amino acid residues identical to $S$. cerevisiae at these positions. Interestingly, a single mutation identified in our original analysis of sordarin-resistant alleles in S. cerevisiae was a Y521S substitution that resulted in partial insensitivity to sordarin (Justice et al., 1998). Sequence comparisons show that the sordarin-insensitive Cr. neoformans eEF2 (Fig. 1) diverges further from the sordarin-sensitive Candida species by incorporating changes at positions $519,521,523$ and 524 relative to $S$. cerevisiae eEF2 (Fig. 2 ). Based on these observations, we reasoned that the amino acids corresponding to $S$. cerevisiae eEF2 residues 517-524 define a 'sordarin-specificity region'.
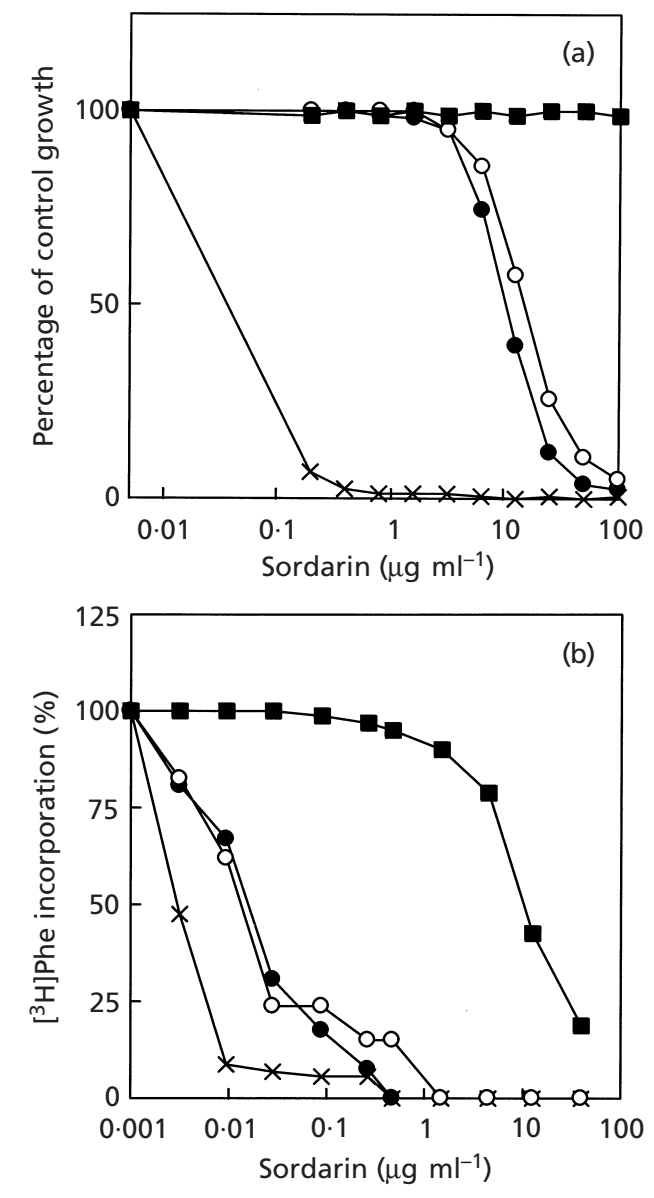

Fig. 3. Identification of residues critical for conferring sordarin specificity to $\mathrm{Ca}$. parapsilosis eEF2. (a) Growth inhibition assays were performed as described in Methods with strains harbouring plasmids that express wild-type Ca. parapsilosis eEF2 or mutant Ca. parapsilosis eEF2s (see Methods for details); $\mathbf{\square}$ wild-type Ca. parapsilosis eEF2; $\bigcirc$, S521Y Ca. parapsilosis eEF2; -, N523S Ca. parapsilosis eEF2; × , S521Y N523S Ca. parapsilosis eEF2. (b) Substitution of amino acid residues 521 and 523 of $\mathrm{Ca}$. parapsilosis eEF2 to those found in S. cerevisiae eEF2 confer in vitro sordarin sensitivity. Translation assays were performed with S. cerevisiae ribosomes and $\mathrm{S} 100$ from $S$. cerevisiae expressing wild-type Ca. parapsilosis eEF2 ( $\mathbf{\square})$, Ca. parapsilosis eEF2 S521Y (O), Ca. parapsilosis eEF2 N523S (0) or Ca. parapsilosis eEF2 S521Y N523S $(\times)$. 
Table 2. Analysis of $S$. cerevisiae substituted eEF2s containing the 'sordarin-specificity region' from different species

S. cerevisiae substituted eEF2s containing the 'sordarin-specificity region' from different fungal species or human eEF2 were constructed as described in Methods. $\mathrm{IC}_{50}$ values for strains harbouring these constructs were then obtained from growth inhibition assays and in vitro translation assays as described in Methods. The values are a mean of three independent assays.

\begin{tabular}{|c|c|c|c|}
\hline \multirow{2}{*}{$\begin{array}{l}\text { Source of 'sordarin- } \\
\text { specificity region' }\end{array}$} & \multirow[t]{2}{*}{ Amino acid sequence } & \multicolumn{2}{|c|}{ Sordarin $\mathrm{IC}_{50}\left(\mu \mathrm{g} \mathrm{ml}^{-1}\right)$} \\
\hline & & $\begin{array}{l}\text { Growth } \\
\text { inhibition }\end{array}$ & $\begin{array}{c}\text { In vitro } \\
\text { translation }\end{array}$ \\
\hline S. cerevisiae & P C V L T Y M S E S & $0 \cdot 5$ & $0 \cdot 006$ \\
\hline Ca. glabrata & P C V L T $\varrho$ M S E S & $4 \cdot 0$ & $0 \cdot 050$ \\
\hline Ca. guilliermondii & P C V $\underline{K} T Y M S E S$ & $0 \cdot 6$ & $0 \cdot 010$ \\
\hline Ca.krusei & P C V L T Y M S $\underline{P} S$ & $>100$ & - \\
\hline Ca. Insitaniae & P C V L T $\underline{S} I N E S$ & $>100$ & $>100$ \\
\hline Ca. parapsilosis & P C V L T $\underline{S} M \underline{N} E S$ & $>100$ & $>100$ \\
\hline Cr. neoformans & $\mathrm{P} C \mathrm{~V} \underline{\mathrm{K}} \mathrm{T} \underline{\mathrm{W}} \mathrm{M} \underline{\mathrm{G}} \mathrm{D} \mathrm{S}$ & $35 \cdot 0$ & $0 \cdot 035$ \\
\hline Human & $\mathrm{P} \underline{\mathrm{M}} \mathrm{V} \underline{\mathrm{Q}} \underline{\mathrm{C}} \underline{I} \underline{\mathrm{E}} \mathrm{ES}$ & $>100$ & $>100$ \\
\hline
\end{tabular}

To test our hypothesis, we substituted two amino acid residues, S521 and N523, in the naturally sordarininsensitive $\mathrm{Ca}$. parapsilosis eEF2 with the corresponding amino acids, Y521 and S523, respectively, found in $S$. cerevisiae eEF2. The modified Ca. parapsilosis eEF2 was transformed into $S$. cerevisiae and assayed for functional complementation and sordarin sensitivity. The results show that the S521Y N523S doubly substituted $\mathrm{Ca}$. parapsilosis eEF2 is sensitive to sordarin while wild-type Ca. parapsilosis eEF2 is completely insensitive to sordarin at our test levels (Fig. 3a). These findings are consistent with the in vitro translation data using S521Y N523S substituted Ca. parapsilosis eEF2 (Fig. 3b). Similar results are observed in the corollary experiment with Y521S and S523N substituted S. cerevisiae eEF2 (see Table 2). This demonstrates that amino acids at positions 521 and 523 are the critical residues in conferring sordarin insensitivity to $\mathrm{Ca}$. parapsilosis eEF2.

To assess the relative contribution of the individual amino acids at positions 521 and 523, we replaced each residue in $\mathrm{Ca}$. parapsilosis eEF2 with the corresponding amino acid from $S$. cerevisiae eEF2. These two constructs were expressed in S. cerevisiae independently and sordarin $\mathrm{IC}_{50}$ values were determined in both growth inhibition and in vitro translation assays (Fig. 3a, b). The single amino acid substitutions in Ca.parapsilosis eEF2 conferred partial resistance $\left(\mathrm{IC}_{50}\right.$ of $10-50 \mu \mathrm{g} \mathrm{ml}^{-1}$ ) to sordarin, in contrast to the complete sensitivity of the Ca. parapsilosis eEF2 in which both the residues had been changed. The $\mathrm{IC}_{50}$ values for the individual substitutions suggest that amino acids 521 and 523 may play a synergistic role in conferring sordarin resistance to Ca. parapsilosis eEF2.

The results described above underscore the importance of residues 521 and 523 in determining sordarin spe- cificity in Ca. parapsilosis. Other species of Candida which are not sensitive to sordarin do have Tyr and Ser at positions 521 and 523 in their eEF2s, respectively (Fig. 2), but differ at positions 519 (Ca. guilliermondii) or 524 (Ca. krusei). Cr. neoformans eEF2, which is partially insensitive to sordarin, differs from $S$. cerevisiae eEF2 at positions corresponding to residues $519,521,523$ and 524. To further evaluate the role of these residues in conferring sordarin specificity in the context of $S$. cerevisiae eEF2, we created a set of substituted $S$. cerevisiae eEF2s by replacing amino acids between 517 and 524 with residues from the corresponding region in either Ca. glabrata, Ca. guilliermondii, Ca. krusei, Ca. lusitaniae, Ca. parapsilosis or $\mathrm{Cr}$. neoformans eEF2 (Table 2). In addition, to assess the contribution of individual amino acids in conferring sordarin's selectivity, we generated a set of substituted S. cerevisiae eEF2 constructs that differed from each other by a single amino acid in this region (Table 3). All eEF2 constructs were assayed for sordarin sensitivity. As seen in Table 3, S. cerevisiae eEF2s substituted with residues found in either Ca. parapsilosis, Ca. lusitaniae or Ca. krusei are fully insensitive to sordarin whereas the mutants with $\mathrm{Ca}$. glabrata, $\mathrm{Ca}$. guilliermondii or $\mathrm{Cr}$. neoformans residues are partially sensitive $\left(\mathrm{IC}_{50} \quad 0 \cdot 6-35 \mu \mathrm{g} \mathrm{ml}^{-1}\right)$. The data also suggest that individual residues may play a synergistic role in sordarin's selectivity; e.g. single amino acid substitutions between 517 and 524 from $\mathrm{Cr}$. neoformans eEF2 result in little resistance to sordarin whereas multiple substitutions result in an $S$. cerevisiae eEF2 that is quite insensitive (up to 800-fold) to sordarin (see Tables 2 and 3 ). Moreover, the identity of the amino acid substitution at a given position determines the extent to which the eEF2 construct becomes insensitive to sordarin. For example, the E524P substituted $S$. cerevisiae eEF2 (as seen in Ca.krusei $\mathrm{eEF} 2$ ) is completely insensitive to sordarin, while E524D (characteristic of 
Table 3. Sordarin sensitivity of substituted S. cerevisiae eEF2s

$\mathrm{IC}_{50}$ values for the substituted $S$. cerevisiae eEF2s were obtained from growth inhibition assays and in vitro translation assays, where performed, as described in Methods. Only changes showing significant shifts upwards in sensitivity were examined by in vitro translation assays. The values are a mean of three independent assays. This table includes single amino acid substitutions in

S. cerevisiae (S.c.) eEF2 with amino acid residues from Ca. glabrata (C.g.), Ca. lusitaniae (C.1.), Ca. parapsilosis (C.p.), Ca. krusei (C.k.), Cr. neoformans (C.n.) and human (h) eEF2s in the 'sordarinspecificity region', in addition to the alanine substitutions between amino acids 517 and 525 of S. cerevisiae eEF2. S. cerevisiae and C. albicans are identical in the 'sordarin-specificity region'.

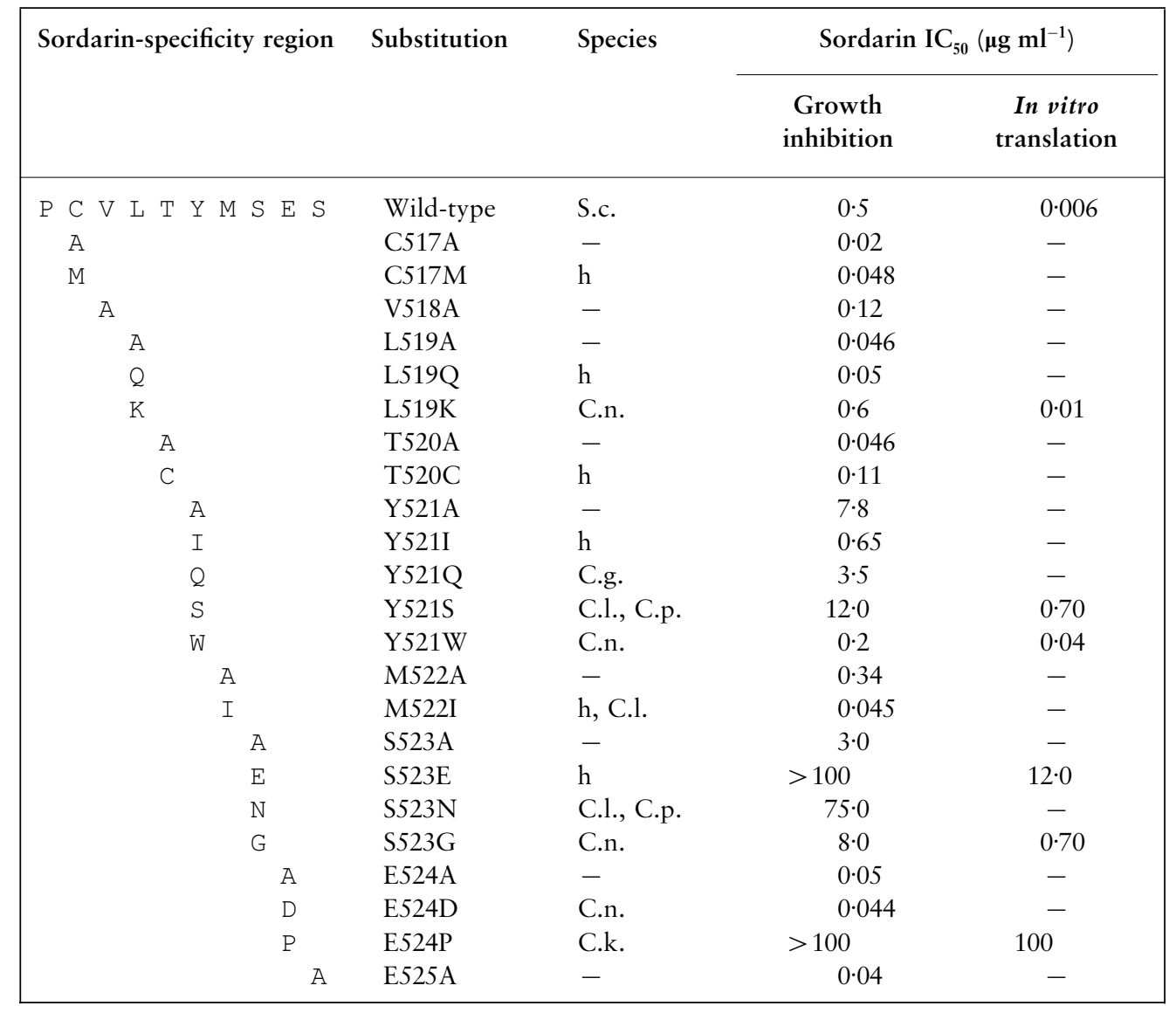

Cr. neoformans eEF2) has no significant effect on sordarin sensitivity in whole-cell assays (Table 3).

To further examine the contribution of the individual residues to sordarin's selectivity, we performed alaninescanning mutagenesis of the 'sordarin-specificity region' by replacing individual amino acids in this region with alanine. Alanine-scanning mutagenesis allows a putative functional side chain to be removed without perturbing the backbone structure, thus enabling the role of the side chain to be investigated without gross changes in protein structure. All nine alanine mutants tested were able to functionally complement a $\Delta$ eft $1 \Delta$ eft 2 S. cerevisiae strain, suggesting that amino acids in the "sordarinspecificity region' are not essential for the function of eEF2. However, when assayed for the sordarin-resistance phenotype, only the Y521A and S523A substituted eEF2s showed some degree of sordarin insensitivity (Table 3), corroborating our results that residues at 521 and 523 in eEF2 play a crucial role in determining sordarin sensitivity.

\section{Human eEF2 and sordarin insensitivity}

Earlier studies revealed that sordarin had no effect on protein synthesis in mammals and plants despite the fact that the soluble translation factors are highly conserved within eukaryotes (Justice et al., 1998). To investigate the role of mammalian eEF2 in sordarin insensitivity, we aligned the deduced protein sequence of human eEF2 with that of the fungal eEF2s. Comparison of human and fungal eEF2 proteins showed that although there are highly conserved regions, there are also conspicuous differences, including some in the "sordarin-specificity region' corresponding to $S$. cerevisiae residues 517-524 (Fig. 2). To examine whether this region contributes to the lack of sordarin sensitivity of mammalian eEF2, a substituted $S$. cerevisiae eEF2 with amino acids $517-524$ 
replaced by the corresponding residues from human eEF2 was generated and assayed for growth inhibition in sordarin-containing medium. As seen in Table 2, the wild-type $S$. cerevisiae eEF2 is sensitive to sordarin, whereas the substituted $S$. cerevisiae eEF2 is fully insensitive to the compound. This result, taken together with the results described above, demonstrates that the identity of eEF2 residues $517-524$ is sufficient to account for sordarin's fungal specificity.

S. cerevisiae eEF2 differs from human eEF2 at six positions in the 'sordarin-specificity region'. We examined the role of these individual residues in conferring sordarin insensitivity with mutant $S$. cerevisiae eEF2 constructs that had single amino acid replacements from the human eEF2 between residues 517 and 524. The results from growth inhibition studies showed that a single substitution at position 523 to the corresponding amino acid from the human eEF2 rendered the $S$. cerevisiae construct insensitive to sordarin while the change at 521 from Tyr to Ile conferred partial insensitivity (Table 3). Amino acid substitutions at the other positions in this region did not significantly change the efficacy of sordarin. The results clearly suggest that the eight-amino-acid region of human eEF2 is sufficient to confer sordarin insensitivity on $S$. cerevisiae that is equal to that observed with mammalian eEF2 (see Fig. 1). Similar to the fungal eEF2s, the amino acid corresponding to $S$. cerevisiae residue 523 in human eEF2 is the major determinant of sordarin efficacy.

\section{DISCUSSION}

Based on data obtained from analysis of the fungal and human eEF2 proteins, we conclude that the amino acid residues corresponding to positions 521, 523 and 524 of S. cerevisiae eEF2 are the major determinants of sordarin specificity found in nature. Our results show that despite the extensive similarity between fungal and mammalian protein synthesis factors, unique single amino acid differences in highly conserved proteins such as eEF2 can provide the required selectivity for identifying potent therapeutic compounds.

In this paper, we demonstrate that the fungal selectivity of sordarin can be mapped to an eight-amino-acid region within eEF2. To show this, heterologous fungal eEF2s and S. cerevisiae substituted eEF2s were expressed in $S$. cerevisiae. The observation that eEF2s from several Candida species were able to functionally complement $S$. cerevisiae eEF2 was not unexpected since this had been previously shown with eEF2 from $\mathrm{Ca}$. albicans (Mendoza et al., 1999). However, we were surprised to find that the highly related fungal eEF2s from $\mathrm{Ca}$. tropicalis and $\mathrm{Cr}$. neoformans could not functionally substitute for $S$. cerevisiae eEF2. Interestingly, we also found that $\mathrm{Ca}$. tropicalis eEF2 exhibited a dominant negative lethal phenotype when expressed in S. cerevisiae (M. Shastry and others, unpublished results). These findings suggest that there are critical determinants for species-specific function of eEF2. The vast majority of data describing the interaction of elongation factors with the ribosome come from studies done in prokaryotes. For example, it has been shown that the eukaryotic translocase eEF2 does not function with the prokaryotic $70 \mathrm{~S}$ ribosome and conversely the prokaryotic EF-G is not functional in the context of the $80 \mathrm{~S}$ ribosome (Grasmuk et al., 1977; Taira et al., 1972). In a recent study, Uchiumi et al. (1999) swapped the L8 complex consisting of L7/L12 and L10 proteins in E. coli with its counterpart from rat, comprising P1, P2 and P0 proteins, and showed that the hybrid ribosome displayed eEF2-dependent but not EF-G-dependent GTPase activity. This hybrid system demonstrates that the stalk protein complex determines the specificity of the ribosome-translocase interaction. These results correlate with our unpublished data (M. C. Justice and others) showing that some combinations of sordarin-resistant alleles of EFT2 and $P O$ are synthetically lethal while others result in a synergistic effect on sordarin resistance. Uchiumi et al. (1999) also showed that the 23S rRNA plays a vital role in this interaction. Further, cryoelectron microscopy studies of the eukaryotic ribosome-eEF2 complex stabilized by sordarin suggest that the interaction between the eEF2 and stalk region of the large subunit is more extensive than in prokaryotes (Gomez-Lorenzo et al., 2000). These results underscore the close interplay between the ribosomal stalk complex, elongation factor 2 and the rRNA during translocation.

Taken together, these observations suggest that there are critical interactions between ribosomal proteins and eEF2 that are important for species-specific expression of eEF2. Despite a wealth of biochemical data describing interactions between EF-G and the ribosome, there is relatively little known about the specific interactions of eEF2 and the ribosome. The reagents produced in this study should prove useful for identification of critical determinants of eEF2 function and interactions with other macromolecules in the translational apparatus.

\section{ACKNOWLEDGEMENTS}

We would like to thank Dr Terri Goss Kinzy of UMDNJ Robert Wood Johnson Medical School, Piscataway, NJ, for reading the manuscript and helpful discussions.

\section{REFERENCES}

Capa, L., Mendoza, A., Lavandera, J. L., Gomez de las Heras, F. \& Garcia-Bustos, J. F. (1998). Translation elongation factor 2 is part of the target for a new family of antifungals. Antimicrob Agents Chemother 42, 2694-2699.

Dominguez, J. M. \& Martin, J. J. (1998). Identification of elongation factor 2 as the essential protein targeted by sordarins in Candida albicans. Antimicrob Agents Chemother 42, 2279-2283.

Dominguez, J. M., Kelly, V. A., Kinsman, O. S., Marriott, M. S., Gomez de las Heras, F. \& Martin, J. J. (1998). Sordarins: a new class of antifungals with selective inhibition of the protein synthesis elongation cycle in yeasts. Antimicrob Agents Chemother 42, 2274-2278.

Dominguez, J. M., Gomez-Lorenzo, M. G. \& Martin, J. J. (1999). Sordarin inhibits fungal protein synthesis by blocking translocation differently to fusidic acid. J Biol Chem 274, 22423-22427. 
Gomez-Lorenzo, M. G. \& Garcia-Bustos, J. F. (1998). Ribosomal Pprotein stalk function is targeted by sordarin antifungals. $\mathrm{J} \mathrm{Biol}$ Chem 273, 25041-25044.

Gomez-Lorenzo, M. G., Spahn, C. M. T., Agrawal, R. K. \& 7 other authors (2000). Three-dimensional cryo-electron microscopy localization of EF2 in the Saccharomyces cerevisiae 80S ribosome at $17 \cdot 5 \AA$ A resolution. EMBO J 19, 2710-2718.

Grasmuk, H., Nolan, R. D. \& Drews, J. (1977). Interchangeability of elongation factor-Tu and elongation factor-1 in aminoacyltRNA binding to $70 \mathrm{~S}$ and $80 \mathrm{~S}$ ribosomes. FEBS Lett 82, 237-242.

Hauser, D. \& Sigg, H. P. (1971). Isolation and decomposition of sordarin. Helv Chim Acta 54, 1178-1190.

Herreros, E., Martinez, C. M., Almela, M. J., Marriott, M. S., De Las Heras, F. G. \& Gargallo-Viola, D. (1998). Sordarins: in vitro activities of new antifungal derivatives against pathogenic yeasts, Pneumocystis carini and filamentous fungi. Antimicrob Agents Chemother 42, 2863-2869.

Ho, S. N., Hunt, H. D., Horton, R. M., Pullen, J. K. \& Pease, L. R. (1989). Engineering hybrid genes without the use of restriction enzymes: gene splicing by overlap extension. Gene 77, 51-59.

Justice, M. C., Hsu, M.-J., Tse, B., Ku, T., Balkovec, J., Schmatz, D. \& Nielsen, J. (1998). Elongation factor 2 as a novel target for selective inhibition of fungal protein synthesis. J Biol Chem 273, 3148-3151.

Justice, M. C., Ku, T., Hsu, M.-J., Carniol, K., Schmatz, D. \& Nielsen, J. (1999). Mutations in ribosomal protein L10e confer resistance to the fungal-specific eukaryotic elongation factor 2 inhibitor sordarin. J Biol Chem 274, 4869-4875.

Mendoza, A., Serramia, M. J., Capa, L. \& Garcia-Bustos, J. F. (1999). Translation elongation factor 2 is encoded by a single essential gene in Candida albicans. Gene 229, 183-191.

Phan, L. D., Perentesis, J. P. \& Bodley, J. W. (1993). Saccharomyces cerevisiae elongation factor 2. Mutagenesis of the histidine precursor of diphthamide yields a functional protein that is resistant to diphtheria toxin. J Biol Chem 268, 8665-8668.

Prodromou, C. \& Pearl, L. H. (1992). Recursive PCR: a novel technique for total gene synthesis. Protein Eng 5, 826-829.

Taira, H., Ejiri, S. \& Shimura, K. (1972). The interaction of elongation factor 2 with ribosomes from silk gland. Formation of an EF-2-ribosome-GDP complex. J Biochem (Tokyo) 72, 1527-1535.

Thompson, J. D., Higgins, D. G. \& Gibson, T. J. (1994). ClustaL w : improving the sensitivity of progressive multiple sequence alignment through sequence weighting, position-specific gap penalties and weight matrix choice. Nucleic Acids Res 22, 4673-4680.

Uchiumi, T., Hori, K., Nomura, T. \& Hachimori, A. (1999). Replacement of L7/L12.L10 protein complex in Escherichia coli ribosomes with the eukaryotic counterpart changes the specificity of elongation factor binding. J Biol Chem 274, 27578-27582.

Received 20 July 2000; revised 4 October 2000; accepted 9 October 2000. 